\title{
Ensayos experimentales y análisis numérico de la estructura del material compuesto alternativo para la reparación de superficies de vuelo en aeronaves
}

\section{(Experimental tests and numerical analysis of the structure of the alternative composite material for the repair of flight surfaces in aircraft)}

\author{
César Arroba Arroba', Manuel Telenchana Flores², Juan Paredes Salinas³, Salomón Fiallo Ortega ${ }^{4}$ \\ y Henry Vaca Ortega ${ }^{5}$
}

\begin{abstract}
Resumen
En la actualidad, los materiales compuestos de matriz polimérica son utilizados en múltiples aplicaciones, una de las áreas más importantes de aplicación es en el sector aeronáutico, debido a la relativamente baja densidad que tienen los materiales combinados. Estos compuestos tienen buenas propiedades mecánicas, comparables con materiales metálicos, pero que aportan beneficios adicionales. El objetivo de este trabajo es hacer los ensayos experimentales y el análisis numérico de la estructura del material compuesto alternativo para emplearlos en la reparación de las superficies de vuelo de aeronaves del CIDFAE. Se manejó un proceso ampliamente extendido de envasado al vacío (vacuum bagging) para obtener un compuesto más homogéneo, Lo que redujo los defectos en el procesamiento, y controló mejor la fracción de las fibras y el espesor global del compuesto. Se elaboraron probetas de resina epóxica reforzada con capas de tejido plano de tres fibras distintas de: carbono, vidrio y aramida, en diferentes orientaciones, que fueron ensayadas a tracción, flexión e impacto para determinar sus propiedades mecánicas. Se ha demostrado que los resultados conseguidos mediante la metodología analítica y el método de elementos finitos son aproximados a los resultados que se obtienen con ensayos destructivos. Los resultados experimentales muestran que los valores de resistencia a la tracción son mayores en los materiales de resina epóxica reforzados con 4 capas de fibra de carbono en orientación $0^{\circ}-90^{\circ}$.
\end{abstract}

\section{Palabras clave}

Superficie de vuelo, compuesto, reparación, aeronaves, configuración.

\begin{abstract}
Nowadays, polymeric matrix composite materials are used in multiple applications. One of the important areas of application is in the aeronautical sector, due to the relatively low density of the combined materials; these compounds have good mechanical properties, comparable to metallic materials, but providing additional benefits. The objective of this work is to carry out the experimental tests and the numerical analysis of the structure of the alternative composite material to be used to repair of the flight surfaces of CIDFAE aircraft. A widely extended vacuum bagging process was used to obtain a more homogeneous compound, thus reducing defects in processing, better controlling the fiber fraction and the overall thickness of the compound. Specimens of epoxy resin reinforced with layers of flat fabric of three different fibers of: carbon, glass and aramid, in different orientations, were made, which were tested for traction, bending and impact to determine their mechanical properties. It has been shown that the results obtained by analytical methodology and the finite element method are approximate to the results obtained with destructive tests. The experimental results show that the tensile strength values are higher in epoxy resin materials reinforced with 4 layers of carbon fiber in orientation $0^{\circ}-90^{\circ}$.
\end{abstract}

\section{Keywords}

Flight surface, compound, repair, aircraft, configuration.

Universidad Técnica de Ambato (UTA), Ambato, Ecuador [ch.arroba@uta.edu.ec, https://orcid.org/0000-0001-5656-7549]. UTA, Ambato, Ecuador [davidtelenchana@gmail.com, https://orcid.org/0000-0002-0337-1099].

UTA, Ambato, Ecuador [jgparedesauta.edu.ec, https://orcid.org/0000-0002-3118-9323].

Centro de Investigación de la Fuerza Aérea Ecuatoriana, Quito, Ecuador [sfiallos@afae.mil.ec, https://orcid.org/0000-00030047-2289].

5 UTA, Ambato, Ecuador [whvaca@uta.edu.ec, https://orcid.org/0000-0003-4321-5864]. 


\section{Introducción}

En la actualidad, las fibras de carbono, las de vidrio y las de Kevlar, consideradas fibras sintéticas de alto rendimiento, se utilizan extensamente en la fabricación de estructuras aeronáuticas; estas fibras sintéticas forman elementos estructurales concretos. No obstante, debido al incremento de la conciencia ecológica, varias investigaciones se han enfocado en emplear compuestos para reparar componentes aeronáuticos, evitando desechar en su totalidad un elemento deteriorado. Las empresas líderes en la fabricación de aviones como Airbus y Boeing investigan sobre el uso de fibras alternativas. Las fibras alternativas tienen propiedades mecánicas similares en combinación con la matriz del compuesto, baja densidad, bajo costo y son capaces de ser empleadas para reemplazar materiales tradicionales (Arockiam et al., 2018).

La utilización de materiales compuestos con refuerzos de fibras ha contribuido en la fabricación de aeronaves, especialmente en las superficies de vuelo. Varias investigaciones proponen considerar materiales alternativos para su uso en aeronaves, estas aeronaves tienen estrictas necesidades de seguridad, por lo que los materiales por emplear se someten a un control estricto (Zhu et al., 2020; Zimmermann \& Wang, 2020). Adicional a un proceso de fabricación, es necesario tener alternativas de configuración y fabricación del material para reparar los componentes de las aeronaves.

Un método relativamente reciente de reparación de componentes de aeronaves que presentan agrietamientos o daños es el de reparación con parches de material compuestos (Wang et al., 2019). Este procedimiento promueve una técnica de reparación innovadora que muestra el potencial de las fibras naturales como materiales viables para reparaciones aeronáuticas y hasta aeroespaciales, ya que brindan tanto un ahorro de coste como disminuyen la densidad, en comparación con otras fibras empleadas (Khirul et al., 2018). Además, hay que entender que la capacidad de resistencia de la reparación mejora con la orientación de la fibra a través de un análisis numérico de las capas de fibra en estudio (Jiang et al., 2019).

El método de reparación con parches de material compuesto es empleado por el Centro de Investigación y Desarrollo de la Fuerza Aérea Ecuatoriana (CIDFAE) en la reparación de algunas superficies de vuelo que presentan daños. Después de la reparación, existe incertidumbre sobre la resistencia que proporciona en el componente, por esto es necesario conocer cuál es la resistencia que debe proporcionar cada una de las capas que conforman el material compuesto aplicado.

En los últimos años, los polímeros han influido en la tecnología más que cualquier otro material, y su combinación con fibras se asocia con propiedades comparables con materiales metálicos, que son reemplazados por los compuestos en aplicaciones estructurales y funcionales. La mejora de las propiedades de un compuesto, mediante el control de sus componentes individuales, cumple con requisitos que colocan al material compuesto como una alternativa de uso en aplicaciones militares, aeroespaciales, comerciales actuales, entre otras (Siddiquee et al., 2020).

Los materiales compuestos pueden definirse como la combinación a un nivel microscópico de uno o más componentes, que no son solubles entre ellos, de la que se obtiene un nuevo material con propiedades determinadas que dependen del tipo de interfaz y de las características de sus constituyentes (Kar, 2017). Sin embargo, el material compuesto formado muestra propiedades únicas no vistas en los componentes individuales. Este material se forma generalmente de una fase rígida y resistente, denominada refuerzo, que se encuentra embebido en un material continuo denominado matriz, encargado de repartir y transmitir las cargas del refuer- 
zo. Una selección cuidadosa tanto de refuerzo como de matriz produce propiedades mecánicas especiales en el compuesto (Kar, 2017; Picu \& Ganghoffer, 2020).

Dentro de las diferentes clasificaciones que se puede hacer de los materiales compuestos, quizás, la más significativa sea la que se refiere al tipo de matriz y refuerzo empleado. Además, las propiedades del material compuesto dependen también de cómo el compuesto ha sido fabricado, por lo que se tiene una amplia gama de comportamientos mecánicos por cada estructura particular producida, lo cual diversifica las aplicaciones de uno u otro compuesto (Kar, 2017; Picu \& Ganghoffer, 2020). Los materiales, según el tipo de matriz, se pueden clasificar en matriz metálica, cerámica y polimérica. Según el tipo de refuerzo, se pueden clasificar en materiales reforzados con partículas, fibras y refuerzos estructurales (Chawla, 2019; Pero-Sanz Elorz et al., 2019).

Durante la fabricación se emplea un proceso de envasado al vacío (vacuum bagging). Este proceso proporciona un alto refuerzo, una mejor adherencia entre los componentes del compuesto y mayor control del porcentaje de volumen de fibra en comparación con los procesos manuales (Kar, 2017). En el campo de los polímeros ya se ha empleado procesos de moldeo asistidos por vacío en los que se mejora significativamente la concentración del polímero en el proceso (Suzuki et al., 2017). En el procesamiento de materiales compuestos con una base de fibras de carbono, a diferentes disposiciones, se ha conseguido mejoras en las propiedades mecánicas; los porcentajes de mejora catalogados tiene un valor significativo (Muralidhara et al., 2019).

En el proceso de fabricación aplicado en este trabajo, se procura controlar los parámetros de moldeo, con esto se pretende reducir los defectos en el procesamiento de materiales, en especial sobre los compuestos con fibras sintéticas; adicionalmente, se pretende obtener un espesor homogéneo del compuesto y controlar la fracción de volumen de fibra (Bharath et al., in press; Verma et al., 2020). Hay que considerar que dentro del proceso de envasado al vacío hay que tener especial atención a geometrías complejas como esquinas dentro del moldeo del material, la presión es un factor determinante dentro de lo anteriormente mencionado (Levy \& Hubert, 2019).

Esta investigación se enfoca en una matriz termoestable reforzada con fibras sintéticas. Dentro de la industria aeronáutica y aeroespacial, se ha estudiado ampliamente el uso de fibras de carbono, a estos compuestos se los denomina plásticos reforzados con fibra de carbono (o por sus siglas en inglés CFRP); adicional a este tipo de materiales, las fibras que también son empleadas extensamente como refuerzo de los materiales compuestos son las fibras de vidrio y las de aramida (Arockiam et al., 2018; Prasad \& Wanhill, 2017).

Existen diferentes métodos para evaluar las propiedades mecánicas de los materiales compuestos, entre los que se destacan el método experimental, analítico y computacional (Azarafza, 2018; Itou et al., 2017), que ha llevado al desarrollo de herramientas analíticas y computacionales precisas capaces de predecir el comportamiento del material (Boyina et al., 2017). En diversas investigaciones se evalúa el comportamiento de los materiales polímeros reforzados con fibra de carbono; este análisis se hace mediante el método de elementos finitos; además, este estudio se emplea para el análisis de componentes estructurales de aeronaves (Kumar Das \& Roy, 2018, March, 22-24).

La simulación numérica computacional del compuesto logra determinar los efectos de la dirección del refuerzo, después del proceso de formación, por lo que se puede utilizar una simulación que se enfoque en la formación de refuerzos tejidos (Liu et al., 2019; Picu \& Ganghoffer, 2020). El método analítico utiliza expresiones matemáticas para predecir las constantes elásticas del material compuesto como la rigidez y resistencia (Huang et al., 2020). Los métodos 
más utilizados para evaluar las propiedades elásticas de un compuesto es la regla de mezclas (Azarafza, 2018) y la aplicación de la teoría clásica de laminado; estos métodos forman parte del análisis de la micro y macromecánica del compuesto (Krishnadasan et al., 2021).

Los compuestos laminados de fibra se fabrican uniendo dos o más láminas, además, pueden orientarse de tal manera que el componente estructural resultante tenga las características mecánicas deseadas en diferentes direcciones. La dirección influye fuertemente en el comportamiento mecánico del componente que integre el material compuesto en estudio (Picu \& Ganghoffer, 2020; Vasiliev et al., 2017).

En un material compuesto reforzado con fibra, la heterogeneidad del material se debe a sus elementos constituyentes, sin embargo, a una escala característica, se puede tener una buena aproximación de sus propiedades, dichas propiedades mecánicas del material compuesto dependen de las componentes de tensión y deformación. Bajo unas condiciones elásticas y lineales en la que el sólido presenta pequeñas deformaciones, se relacionan mediante la ley de Hooke generalizada, debido a que, a escala macroscópica, el material compuesto puede considerarse homogéneo (Altenbach et al., 2018; Chawla, 2019)

$$
\sigma_{i j}=C_{i j k l} \varepsilon_{k l}
$$

Donde

$\sigma_{i j}$ indica el estado tensional del elemento o componente

$C_{i j k l}$ son las constantes elásticas o rigidez

y $\varepsilon_{k l}$ es la deformación unitaria

La relación deformación-tensión para un material ortotrópico bajo un estado de tensión plana se expresa como la deformación igual a la multiplicación del tensor de flexibilidad multiplicado por el vector de estado tensional:

$$
\left[\begin{array}{c}
\varepsilon_{\mathrm{x}} \\
\varepsilon_{\mathrm{y}} \\
\gamma_{x z}
\end{array}\right]=\left[\begin{array}{ccc}
S_{11} & S_{12} & 0 \\
S_{12} & S_{22} & 0 \\
0 & 0 & S_{66}
\end{array}\right]\left[\begin{array}{c}
\sigma_{\mathrm{x}} \\
\sigma_{\mathrm{y}} \\
\tau_{x y}
\end{array}\right]
$$

Proporcionadas las características geométricas de la lámina, se asume un estado de tensión plana. La relación deformación-tensión para una lámina unidireccional se expresa en términos de matriz de rigidez reducida como:

$$
\left[\begin{array}{c}
\sigma_{1} \\
\sigma_{2} \\
\tau_{12}
\end{array}\right]=\left[\begin{array}{ccc}
\frac{E_{1}}{1-v_{12} v_{21}} & \frac{v_{21} E_{1}}{1-v_{12} v_{21}} & 0 \\
\frac{v_{12} E_{2}}{1-v_{12} v_{21}} & \frac{E_{2}}{1-v_{12} v_{21}} & 0 \\
0 & 0 & G_{12}
\end{array}\right]\left[\begin{array}{c}
\varepsilon_{1} \\
\varepsilon_{2} \\
\gamma_{12}
\end{array}\right]
$$

Donde $v_{21}=\left(\frac{E_{2}}{E_{1}}\right) * v_{12}$ EC. (4)

Las láminas unidireccionales poseen direcciones preferentes asociadas a la orientación de la fibra del refuerzo o a la simetría de los planos. Debido a estos sistemas de coordenadas 
se utilizan los números 1 y 2 , donde la primera dirección coincide con la dirección de la fibra y la segunda es ortogonal. Para definir los ejes globales o ejes del material $(x, y, z)$, si las direcciones de la fibra no coinciden con las coordenadas globales se puede utilizar la siguiente ecuación.

$$
\left[\begin{array}{c}
\sigma_{1} \\
\sigma_{2} \\
\tau_{12}
\end{array}\right]=T_{\sigma}(\theta)\left[\begin{array}{c}
\sigma_{\mathrm{x}} \\
\sigma_{\mathrm{y}} \\
\tau_{x y}
\end{array}\right]
$$

Donde $T_{\sigma}(\theta)$ se denomina la matriz.

El método de reparación con parches de material compuesto es empleado por el CIDFAE para reparar algunas superficies de vuelo que presentan daños. Después de la reparación, existe incertidumbre sobre la resistencia que proporciona en el componente, por esto es necesario conocer cuál es la resistencia que debe proporcionar cada una de las capas que conforman el material compuesto aplicado.

El objetivo de esta investigación es determinar por separado las propiedades mecánicas del material de cada capa del compuesto utilizado para la reparación de superficies de vuelo, aplicando el método experimental, método analítico y análisis mediante elementos finitos, con el propósito de evaluar cuál es la diferencia entre estos métodos. Además, se espera que estos resultados puedan ser utilizados para diseñar la combinación adecuada del material compuesto que será empleado en las reparaciones de superficies de vuelo en aeronaves.

\section{Metodología}

\section{Materiales y equipos}

En la matriz del compuesto se utilizó resina epoxi de viscosidad media, recomendable para aplicaciones de producción estructural marca Aeropoxy PR2032, combinada con un catalizador PH3660; esta combinación proporciona una humectación excelente de las fibras de vidrio, carbono y aramida utilizadas en presentación de tejido liso. Además, posee aditivos que promueven la adhesión química a las telas hechas con estas fibras.

El refuerzo utilizado fueron las fibras de carbono, vidrio y aramida. Las fibras se presentan en forma de tejido liso. Los materiales y resina fueron proporcionados en el CIDFAE. Las probetas de material compuesto se obtuvieron mediante la estratificación manual combinada con el curado al vacío. El proceso de moldeo se hizo en placa de vidrio de $6 \mathrm{~mm}$ de espesor.

Los ensayos de tracción y flexión se realizaron en la maquina universal de $50 \mathrm{KN}$ para materiales poliméricos, cerámicos, compuestos, Metrotec Serie MTE50. La máquina de impacto por caída de dardos para polímeros se utilizó para el ensayo de impacto.

\section{Metodología experimental}

En la placa de vidrio de $6 \mathrm{~mm}$ se realizó el moldeo abierto para obtener un acabado superficial libre de imperfecciones. Se elaboraron las probetas de material compuesto para ensayos mecánicos de tracción, flexión e impacto, ver Figura 1. 
Figura 1. Fabricación de las probetas

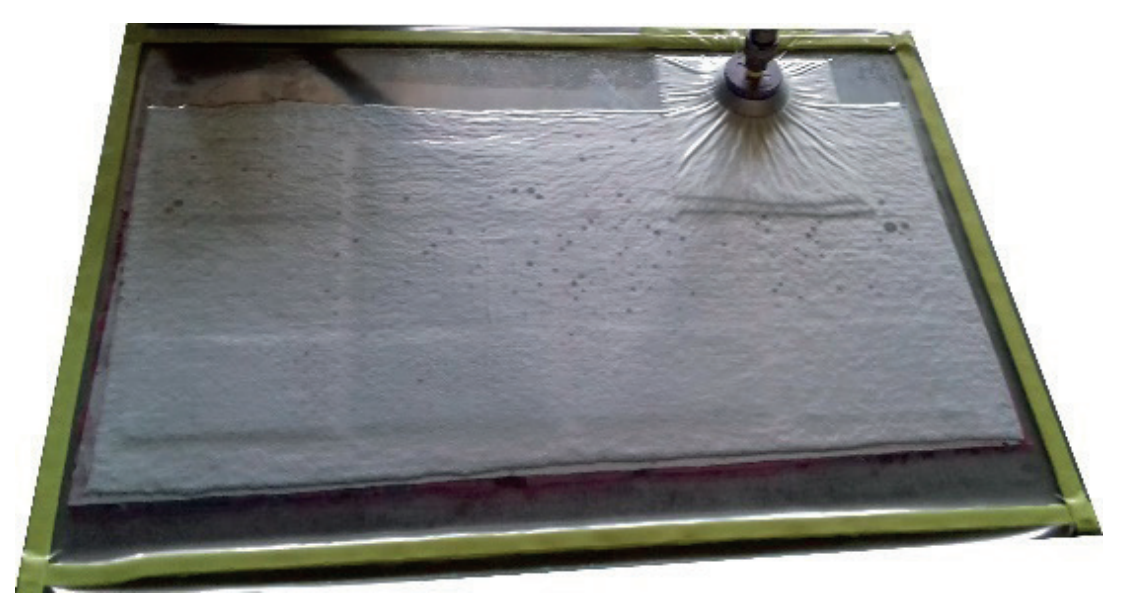

Las dimensiones de la probeta para el ensayo de tracción fueron 250x25xe mm (ASTM D3039/D3039M, 2014), para los de flexión las dimensiones fueron de 160x13xe mm (ASTM D7264/D7264M-07, 2007) y para los de impacto se utilizó la geometría FE de 58 mm de lado (ASTM D5628-96, 1995). El espesor de las probetas está en función del número de capas empleado, para los ensayos de tracción e impacto se utilizó dos capas de fibra sintética, para el de flexión se empleó doce capas por cada fibra sintética.

Tabla 1. Combinaciones del material compuesto para el ensayo de tracción e impacto

\begin{tabular}{|c|c|c|c|c|c|}
\hline \multirow[b]{2}{*}{ Código } & \multicolumn{3}{|c|}{ Combinación } & \multicolumn{2}{|c|}{$\begin{array}{c}\text { Cantidad de probetas } \\
\text { para el ensayo de }\end{array}$} \\
\hline & $\begin{array}{l}\text { Materiales } \\
\text { de refuerzo }\end{array}$ & N. ${ }^{\circ}$ de Capas & Orientación & Tracción & Impacto \\
\hline FV 2C 0-90 & \multirow{4}{*}{ Fibra de vidrio } & \multirow{2}{*}{2 capas } & $0^{\circ}-90^{\circ}$ & 5 & 9 \\
\hline FV 2C 45-135 & & & $45^{\circ}-135^{\circ}$ & 5 & 9 \\
\hline FV 4C 0-90 & & \multirow{2}{*}{4 capas } & $0^{\circ}-90^{\circ}$ & 5 & 9 \\
\hline FV 4C 45-135 & & & $45^{\circ}-135^{\circ}$ & 5 & 9 \\
\hline FC 2C 0-90 & \multirow{4}{*}{ Fibra de carbono } & \multirow{2}{*}{2 capas } & $0^{\circ}-90^{\circ}$ & 5 & 9 \\
\hline FC 2C 45-135 & & & $45^{\circ}-135^{\circ}$ & 5 & 9 \\
\hline FC 4C 0-90 & & \multirow{2}{*}{4 capas } & $0^{\circ}-90^{\circ}$ & 5 & 9 \\
\hline FC 4C 45-135 & & & $45^{\circ}-135^{\circ}$ & 5 & 9 \\
\hline FA 2C 0-90 & \multirow{4}{*}{ Fibra de aramida } & \multirow{2}{*}{2 capas } & $0^{\circ}-90^{\circ}$ & 5 & 9 \\
\hline FA 2C 45-135 & & & $45^{\circ}-135^{\circ}$ & 5 & 9 \\
\hline FA 4C 0-90 & & \multirow{2}{*}{4 capas } & $0^{\circ}-90^{\circ}$ & 5 & 9 \\
\hline FA 4C 45-135 & & & $45^{\circ}-135^{\circ}$ & 5 & 9 \\
\hline \multicolumn{4}{|c|}{ TOTAL } & 60 & 108 \\
\hline
\end{tabular}

El primer paso en la fabricación de las probetas fue el corte de los tejidos de fibra sintética para cada combinación, luego se limpió y aplicó cera en el molde con el fin de facilitar la extracción de las probetas. En la balanza electrónica se pesó la cantidad de masa del refuerzo, y luego se preparó la resina y catalizador con una relación de proporción de 100:27 
en peso y de 3 a 1 en volumen. Seguido de esto, se aplicó la resina en los tejidos de fibra, humectando uniformemente la resina sobre todo el tejido. Se colocó la película de liberación (release film) que cumple la función de dejar pasar el aire y el exceso de resina. Sobre esta se colocó la película de desmolde (peel ply) que deja una textura uniforme en la superficie que no está en contacto con el vidrio. Después se coloca la película de respiración (breather) que cumple la función de absorber el exceso de resina y, finalmente, arriba de todo, se coloca la película de envasado al vacío (vacuum bagging film) con una cinta sellante en los extremos para hacer el vacío. La presión de vacío aplicada fue de -18 inHg durante tres horas y se dejó curar por 24 horas en el molde. Transcurrido ese tiempo, se desmoldó el material compuesto, ver figura 1.

Las combinaciones estudiadas en este trabajo se registraron en la tabla 1 para los ensayos de tracción e impacto y en la tabla 2 para los de flexión.

Tabla 2. Combinaciones del material compuesto: ensayo de flexión

\begin{tabular}{|c|c|c|c|c|}
\hline & \multicolumn{3}{|c|}{ Combinación } & \multirow{2}{*}{$\begin{array}{c}\begin{array}{c}\text { Cantidad } \\
\text { de probetas }\end{array} \\
\text { Flexión }\end{array}$} \\
\hline Ítem & Materiales & \# Capas & Orientación & \\
\hline 1 & Fibra de vidrio & 12 capas & $0^{\circ}-90^{\circ}$ & 5 \\
\hline 2 & Fibra de carbono & 12 capas & $0^{\circ}-90^{\circ}$ & 5 \\
\hline 3 & Fibra de aramida & 12 capas & $0^{\circ}-90^{\circ}$ & 5 \\
\hline \multicolumn{4}{|c|}{ TOTAL } & 15 \\
\hline
\end{tabular}

En el ensayo de tracción se aplicó una carga a una velocidad constante de $5 \mathrm{~mm} / \mathrm{min}$ hasta la carga máxima y mediante inspección visual se determina el modo de falla (ASTM D3039/ D3039M , 2014). El ensayo a flexión se realizó a 1 mm/min de velocidad constante (ASTM D7264/ D7264M-07, 2007). En el ensayo de impacto, el dardo se dejó caer a diferentes alturas, iniciando en $200 \mathrm{~mm}$ para compuestos reforzados de fibra de carbono, $100 \mathrm{~mm}$ para los compuestos de fibra de vidrio y $200 \mathrm{~mm}$ para compuestos reforzados con fibra de aramida; la altura varía hasta determinar la altura de ruptura o fractura por impacto (ASTM D5628-96, 1995).

\section{Metodología para el análisis mediante elementos finitos}

En el análisis de elementos finitos, hecho en ANSYS LS-Dyna Research, se elaboró una geometría tridimensional de 110x25xe mm que simula la zona de aplicación de carga. El análisis fue estático dentro del rango elástico. El espesor y orientación de fibras se configuraron en el módulo ACP (ANSYS Composite PrepPost) según la configuración utilizada para los ensayos experimentales. Las condiciones de frontera de la probeta, indicadas en la Figura 2, se asignaron de acuerdo con el ensayo experimental, colocando en la parte inferior de la probeta restricciones de todos los grados de libertad, mientras que en la cara de la parte superior se aplicaron cargas hasta llegar a la fluencia. 
Figura 2. Condiciones de frontera

\section{B: Static Structural}

Static Structural

Time: 1.s

Force: $2488.5 \mathrm{~N}$

Fixed Support

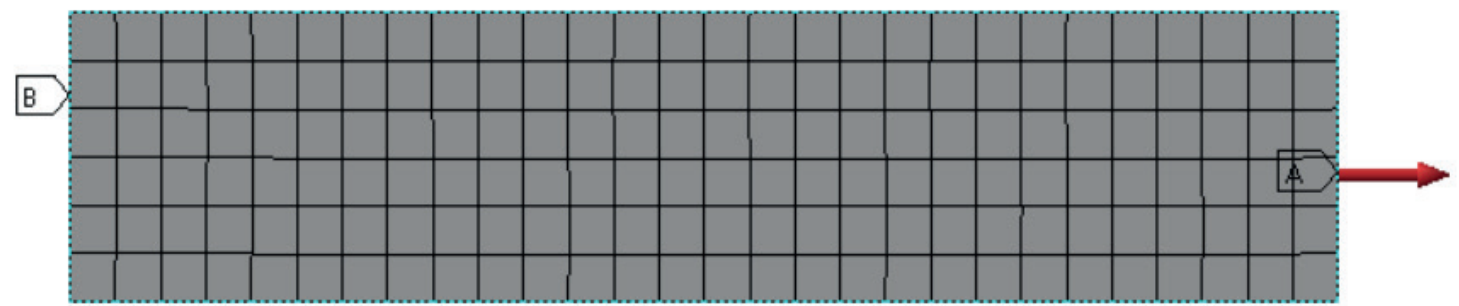

\section{Metodología analítica}

El cálculo del módulo de elasticidad se elaboró a partir del análisis micro y macromecánico empleando los modelos matemáticos presentados en la introducción. Para el cálculo de las constantes elásticas, se utilizarán las propiedades de la tabla 3. Los valores de las fibras de carbono, fibra de aramida y fibra de vidrio se tomaron de Kar (2017) y, finalmente, para la matriz se tomaron los datos de la ficha técnica de la resina.

Tabla 3. Propiedades elásticas de los constituyentes del material compuesto

\begin{tabular}{|c|c|c|c|c|}
\hline Constantes & Fibra de carbono & Fibra de aramida & Fibra de vidrio & Resina epoxi \\
\hline $\mathrm{E}_{1}(\mathrm{Gpa})$ & 230 & 133 & 72 & 2.89 \\
\hline $\mathrm{E}_{2}(\mathrm{Gpa})$ & 24 & 7 & 72 & 2.89 \\
\hline $\mathrm{G}_{12}(\mathrm{Gpa})$ & 50 & 12 & 27.7 & 1.07 \\
\hline $\mathrm{g}_{12}$ & 0.28 & 0.38 & 0.3 & 0.35 \\
\hline
\end{tabular}

A continuación, se detalla un ejemplo utilizando el método analítico para fibra de carbono de dos capas y orientado a $0^{\circ}-90^{\circ}$. Las fracciones volumétricas de la fibra son yf $=0.47$.

El valor de $G_{12}$ de la matriz se calculó mediante la siguiente ecuación (León \& Vásquez, 2010):

$$
G_{m}=\frac{E_{m}}{2 *\left(1+v_{m}\right)}
$$

Se utiliza el subíndice $m$ para propiedades de la matriz y f para propiedades de la fibra; luego, aplicando las ecuaciones de la regla de mezclas se obtiene:

$$
\begin{gathered}
E_{1}=E_{f}{ }^{*} V_{f}+E_{m}{ }^{*}\left(1-V_{f}\right)=230 * 0.47+2.89 *(1-0.47)=109.632 \mathrm{Gpa} \\
E_{2}=\frac{E_{f}{ }^{*} E_{m}}{E_{f}{ }^{*}\left(1-V_{f}\right)+E_{m}{ }^{*} V_{f}}=\frac{230 * 2.89}{230 *(1-0.47)+2.89 * 0.47}=5.393 \mathrm{Gpa} \\
G_{12}=\frac{G_{f}{ }^{*} G_{m}}{G_{f} *\left(1-V_{f}\right)+G_{m} V_{f}}=\frac{50 * 1.07}{50 *(1-0.47)+1.07 * 0.47}=1.982 \mathrm{Gpa}
\end{gathered}
$$




$$
\begin{gathered}
v_{12}=v_{f} * V_{f}+v_{m} *\left(1-V_{f}\right)=0.28 * 0.47+0.35 *(1-0.47)=0.317 \\
v_{21}=\left(\frac{E_{2}}{E_{1}}\right) * v_{12}=(5.393 / 109.632) * 0.317=0.015
\end{gathered}
$$

Si el refuerzo de un compuesto es un tejido en el cual se tiene el mismo número de fibras en la dirección de la urdimbre y de la trama es ortotrópico, la rigidez se reduce drásticamente con respecto a la fibra unidireccional, esto se debe a la flexión de la fibra alrededor de otras fibras para producir el tejido. La pérdida de rigidez es de aproximadamente un 37 \% (León \& Vásquez, 2010).

Para dicha capa, los elementos de la matriz de rigidez se calculan a partir de Vasiliev et al. (2017):

$$
Q_{i j}^{t e j i d o}=\frac{1}{2}\left[\left(\bar{Q}_{i j}\right)_{\theta}+\left(\bar{Q}_{i j}\right)_{-\theta}\right], i, j=1,2,6
$$

Por lo tanto:

$$
\begin{gathered}
\bar{Q}_{0^{\circ}, 90^{\circ}}=\left[\begin{array}{ccc}
110.1766 & 1.7181 & 0 \\
1.7181 & 5.4198 & 0 \\
0 & 0 & 1.982
\end{array}\right] \\
\bar{Q}_{\text {tejido }}=\frac{1}{2}\left[(\bar{Q})_{0^{\circ}}+(\bar{Q})_{90^{\circ}}\right]=\left[\begin{array}{ccc}
57.7982 & 1.7181 & 0 \\
1.7181 & 57.7982 & 0 \\
0 & 0 & 1.982
\end{array}\right]
\end{gathered}
$$

La matriz de flexibilidad transformada es la inversa de $\bar{Q}_{t e j i d o}$ matriz de rigidez:

$$
\bar{S}=\left[\begin{array}{ccc}
0.0173 & -0.0005 & 0 \\
-0.0005 & 0.0173 & 0 \\
0 & 0 & 0.5045
\end{array}\right]
$$

Y, finalmente, las constantes elásticas de la lámina son:

$$
E_{1}=\frac{1}{S_{11}}=57.7471 \mathrm{Gpa} ; E_{2}=\frac{1}{S_{22}}=57.7471 \mathrm{Gpa} ; G_{12}=\frac{1}{S_{66}}=1.982 \mathrm{Gpa} ; v_{12}=-S_{12}{ }^{*} E_{1}=0.029
$$

\section{Resultados}

Los valores de tensión se indican en la Figura 3.1 y corresponden a la resistencia máxima obtenida al aplicar el método de elementos finitos, y se representan en la curva a; el método analítico se representa en la curva b, y el método experimental se representa en la curva c. Los valores del módulo de elasticidad en las direcciones se indica en la Figura 3.2 y corresponde a la resistencia máxima al utilizar el método de elementos finitos (curva a), el método analítico (curva b), y el método experimental (curva c). Los valores de la energía del ensayo de impacto se indican en la Figura 3.3, y corresponden a la resistencia máxima utilizando el método elementos finitos (curva a), y el método experimental (curva b).

El material compuesto reforzado con fibra de carbono y cuatro capas presentó una resistencia a la tracción de aproximadamente $623.305 \mathrm{MPa}$, con un módulo de elasticidad de $80.157 \mathrm{GPa}$, que son los valores más altos de entre todas las combinaciones analizadas. El ensa- 
yo de impacto indica que el material compuesto reforzado con fibra de aramida obtuvo energía de impacto de $1.82 \mathrm{~J}$.

Figura 3. Ensayos realizados según las tres metodologías

\subsection{Resistencia Máxima}

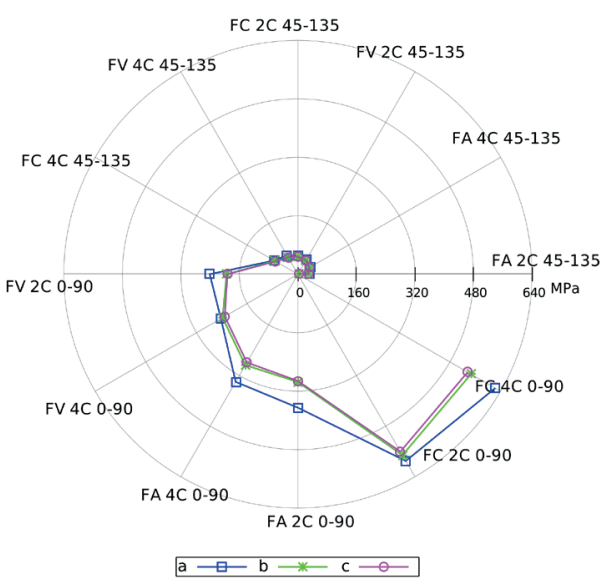

Nota: a. Experimental b. Analítica c. MEF

\subsection{Módulo de Elasticidad E}

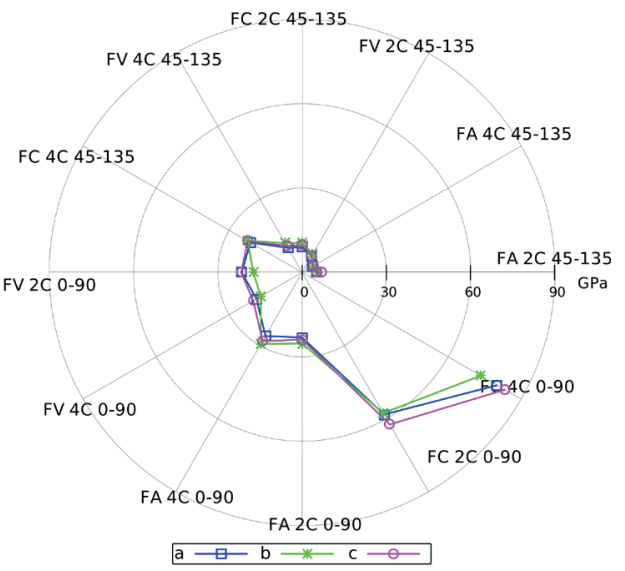

Nota: a. Experimental b. Analítica c. MEF

\subsection{Energía de falla}

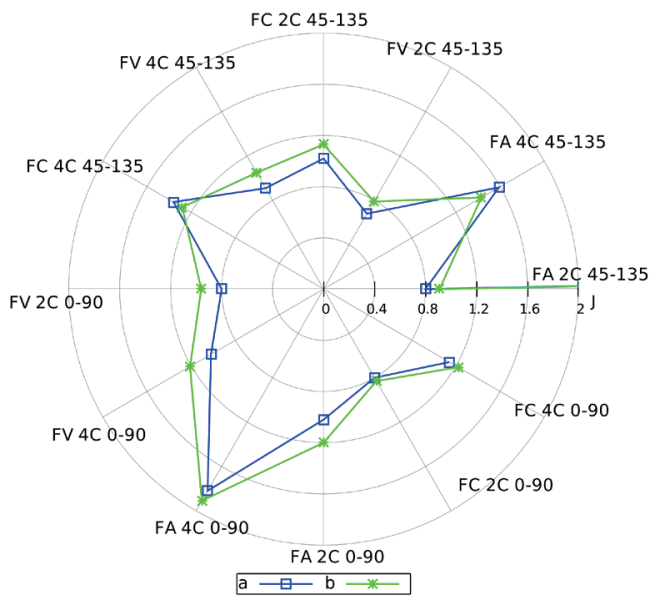

Nota: a. Experimental b. MEF

En el ensayo de flexión de las figuras 4.1 y 4.2 se muestra el esfuerzo máximo y el módulo de flexión. El material compuesto reforzado con fibra de carbono obtuvo valores de 574.10 MPa para el esfuerzo y de 32.201 GPa para el módulo de flexión. 
Figura 4. Ensayos realizados según las tres metodologías

\subsection{Esfuerzo máximo a flexión}

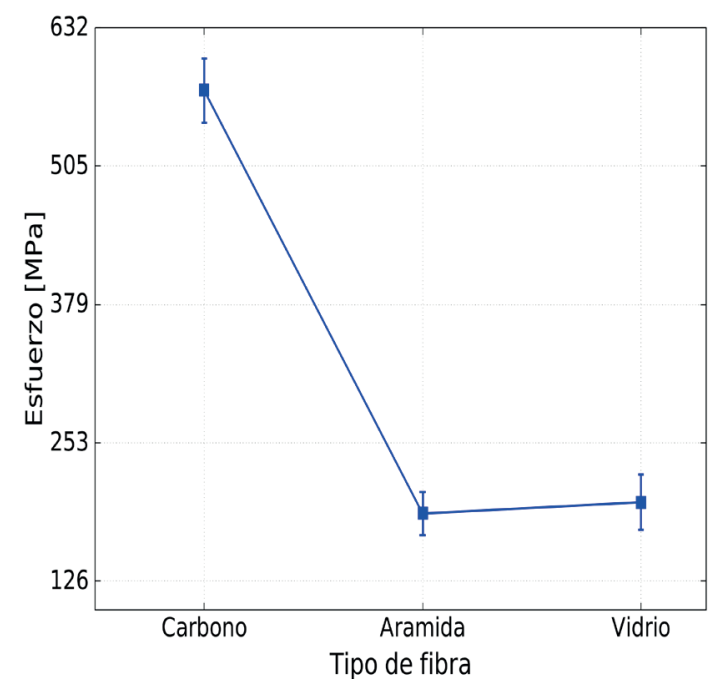

\subsection{Módulo de flexión}

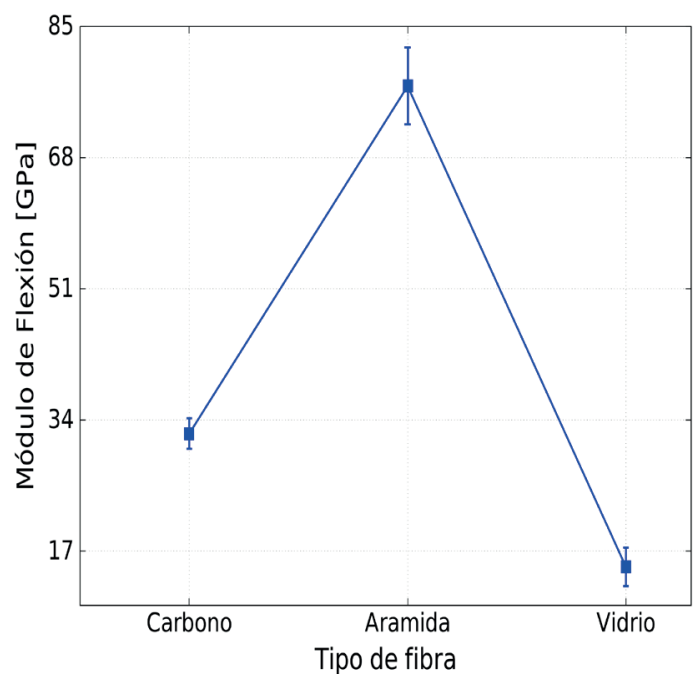

\section{Discusión}

La Figura 5 proporciona mayor claridad para identificar la diferencia de resultados entre las metodologías aplicadas en la determinación de las propiedades del material. La variación de valores fluctúa entre $2 \%$ y $11 \%$ para una misma propiedad, aplicando diferente metodología, estos resultados indican que las metodologías utilizadas en esta investigación para determinar las propiedades del material están muy cercanas a los resultados experimentales.

La combinación de fibras de carbono en la misma dirección de aplicación de la carga presentó la mejor resistencia a la tensión como se muestra en la figura 4.1. La combinación de fibras a $45^{\circ}$ y $135^{\circ}$ independientemente del número de capas reduce, de forma considerable, la resistencia última del material, efecto que también se muestra en los valores del módulo de elasticidad respecto al eje $y$, figura 4.2. Este resultado es importante considerar en el proceso de diseño del material, para ello se debería conocer claramente el estado tensional del elemento o componente $y$, de acuerdo con este estado, disponer de las direcciones de las diferentes fibras. Esto sirve como guía para la selección adecuada de combinaciones de fibras para la reparación de superficies de vuelo de aeronaves, tomando en cuenta la cantidad de capas de fibra sintética y las disposiciones a diferentes ángulos, para obtener un material con características isotrópicas si el estado tensional, debido a la forma y carga del componente, presenta solicitación en los tres ejes globales, o para otro casos donde el estado de carga tenga una dirección preferencial se disponga según la función. 
Figura 5. Variación de resultados para las diferentes metodologías utilizadas para determinar las propiedades del material compuesto

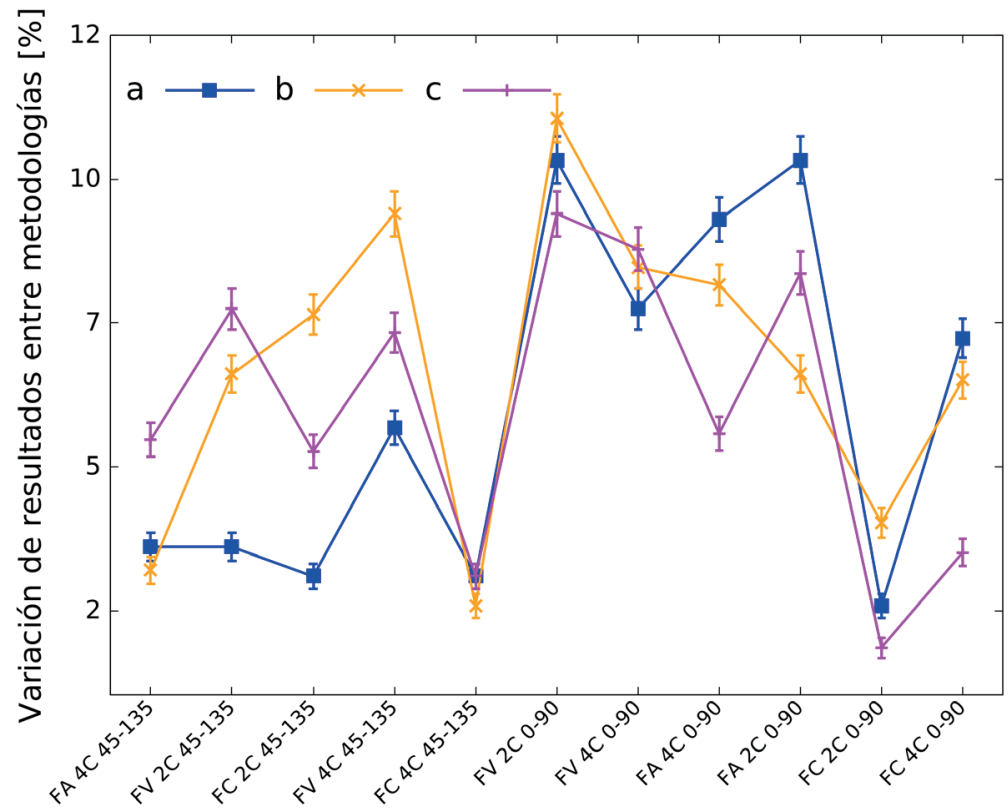

Disposición de capas de fibra

Nota: a. Esfuerzo máximo b. Módulo de elasticidad c. Energía de falla

La figura 3.3 correspondiente al ensayo de impacto, la combinación de fibras de aramida presentó los mejores resultados. El análisis de todos estos resultados indica que en la preparación de un material adecuado para reparar superficies de vuelo es necesario combinar tejidos de fibra de vidrio, de aramida y de carbono. Cada una de estas fibras contribuye en una característica especifica del nuevo material. La fibra de carbono le proporciona una buena resistencia, mientras que la de aramida mejorará la tenacidad, indispensable en eventos de impactos, y la adición de la fibra de vidrio reducirá su costo proporcionará una interfaz volumétrica de resistencia adecuada.

\section{Conclusiones y recomendaciones}

Los ensayos destructivos son muy importantes en el proceso de caracterización del material, sin embargo, se ha demostrado que a través de la metodología analítica y el método de elementos finitos se obtienen resultados muy aproximados. Existe una variación considerable referente a la resistencia a la tracción cuando se cambia el material de refuerzo, número de capas o la orientación de las fibras; la aplicación final, requerimientos estructurales o estado tensional son lo que determinarán la configuración del material compuesto referente al número de capas y orientación. El proceso de manufactura empleado para obtener materiales compuestos se refleja en los resultados finales obtenidos, así pues, será importante desarrollar estudios donde el proceso de fabricación sea distinto.

El proceso de fabricación de envasado al vacío (vacuum bagging) ofrece mejores parámetros de homogeneidad entre matriz y refuerzo; además, se consigue controlar mejor la fracción entre 
las fibras. Con este proceso de fabricación se establece un valor estándar en el espesor del compuesto - que con métodos manuales es difícil de controlar- permitiendo una mejor distribución de esfuerzos en los diferentes puntos de la probeta, lo que ofrece resultados fiables, apegados a la realidad. Esto se relaciona íntimamente con la baja variabilidad existente entre la metodología analítica y el método de elementos finitos con relación a los ensayos destructivos. Se recalca que un parámetro de control riguroso al momento de emplear este proceso es el control de la presión.

Indagar con un análisis de datos de ligue profundamente la relación implícita entre los parámetros de fabricación y los resultados experimentales mediante ensayos destructivos potenciará la perspectiva que se tenga de variar muchas las condiciones de fabricación de manera adecuada, para conseguir un aprovechamiento óptimo del compuesto por fabricar. Adicionalmente, una prueba que cuente con un alto nivel de confianza cuantificable puede ser una alternativa eficaz al momento de optimizar recursos, tanto materiales como investigativos, sabiendo apuntar a un control más preciso de uno u otro factor y descartando los parámetros insignificantes. Una metodología ampliamente empleada cae en el campo de la estadística inferencial por su alto nivel de confianza y su exención aplicada no solo a la industria aeronáutica.

Se recomienda llevar a cabo pruebas de humedad que serán de gran importancia exploratoria en estos materiales compuestos para tener un acercamiento más real a algunas condiciones de trabajo de los elementos en los que se podrían emplear, como la exposición a la intemperie. De la misma forma, las pruebas de inflamabilidad serán fundamentales para obtener datos numéricos frente a posibles accidentes donde se presente fuego. Además, será crucial el estudio de materiales compuestos híbridos que varíen el número de capas y orientación de las fibras a partir de estos refuerzos. Esto permitirá ampliar el campo de conocimiento, ayudará en la selección de materiales y a definir muchas más aplicaciones no solamente dentro de la industria aeronáutica; hay que entender que dentro de esta consideración se toma en cuenta el factor ecológico y la facilidad de contar con un material amigable con el medio ambiente.

\section{Referencias}

Altenbach, H., Altenbach, J., \& Kissing, W. (2018). Mechanics of Composite Structural Elements. Springer. https://doi.org/10.1007/978-981-10-8935-0

Arockiam, N. J., Jawaid, M., \& Saba, N. (2018). 6. Sustainable Bio Composites for Aircraft Components. In M. Jawaid \& M. Thariq (Eds.), Sustainable Composites for Aerospace Applications (109-123). Woodhead Publishing. https://doi.org/10.1016/B978-0-08-102131-6.00006-2

ASTM D3039/D3039M. (2014). Standard Test Method for Tensile Properties of Polymer Matrix Composite Materials D3039. ASTM Standards, 15, 1-13. https://www.astm.org/Standards/D3039

ASTM D5628-96. (1995). Standard Test Method for Impact Resistance of Flat, Rigid Plastic Specimens by Means of a Falling Dart (Tup or Falling Mass). ASTM Book of Standards, 08(October), 1-10. https://www.astm.org/Standards/D5628

ASTM D7264/D7264M-07. (2007). Standard Test Method for Flexural Properties of Polymer Matrix Composite Materials. Annual Book of ASTM Standards, i, 1-11. https://www.astm.org/Standards/D7264

Azarafza, R. (2018). Fabrication, Experimental Modal Testing, and A Numerical Analysis of Composite Sandwich Structures with a Grid-Stiffened Core. Mechanics of Composite Materials, 54(4), 537-544. https://doi.org/10.1007/s11029-018-9762-4

Bharath, D., Sandhya Rani, B., Saritha, V., Irshad Khan, P., \& Kumar Chokka, S. (in press). Tensile and Erosion Behaviour of Medium Calcined Alumina Microparticles on GFRP Composites Fabricated with Vacuum Bagging Process. MaterialsToday: Proceedings, xxxx. https://doi.org/10.1016/j.matpr.2020.08.166 
Boyina, G. R. T., Rayavarapu, V. K., \& Subba Rao, V. V. (2017). Numerical Modelling and Damage Assessment of Rotary Wing Aircraft Cabin Door Using Continuum Damage Mechanics Model. Applied Composite Materials, 24(1), 235-250. https://doi.org/10.1007/s10443-016-9524-1

Chawla, K. K. (2019). Composite Materials. Springer. https://doi.org/10.1007/978-3-030-28983-6

Huang, W., Xie, L., Li, C., Jia, D., Chai, B., Mu, Y., Hou, C., \& Dai, W. (2020). Ananalytical and Experimental Study of T-Shaped Composite Stiffened Panels: Effect of $90^{\circ}$ Plies in Stringers on Curing and Buckling Performance. Applied Composite Materials, 27(5), 597-618. https://doi.org/10.1007/ s10443-020-09816-4

Itou, H., Kimura, M., Chalupecký, V., Ohtsuka, K., Tagami, D., \& Takada, A. (Eds.). (2017). Mathematical Analysis of Continuum Mechanics and Industrial Applications (Vol. 26). Springer. https://doi. org/10.1007/978-981-10-2633-1

Jiang, H., Ren, Y., \& Liu, Z. (2019). Thin-Walled Structures Numerical Prediction for Effects of Fiber Orientation on Perforation Resistance Behaviors of Patch-repaired Composite Panel Subjected to Projectile Impact. Thin Walled Structures, 144(May), 106325. https://doi.org/10.1016/j. tws.2019.106325

Kar, K. K. (Ed.). (2017). Composite Materials. Springer. https://doi.org/10.1007/978-3-662-49514-8

Khirul, M., Muda, H., \& Mustapha, F. (2018). 9. Composite Patch Repair Using Natural Fiber for Aerospace Applications, Sustainable Composites for Aerospace Applications. In M. Jawaid \& M. Thariq (Eds.), Sustainable Composites for Aerospace Applications (171-209). Woodhead Publishing. https://doi.org/10.1016/B978-0-08-102131-6.00009-8

Krishnadasan, C. K., Shanmugam, N. S., Sivasubramonian, B., Nageswara Rao, B., \& Suresh, R. (2021). Analytical Studies and Numerical Predictions of Stresses in Shear Joints of Layered Composite Panels for Aerospace Applications. Composite Structures, 255, 112927. https://doi.org/https:// doi.org/10.1016/j.compstruct.2020.112927

Kumar Das, S., \& Roy, S. (2018, March 22-24). Finite Element Analysis of Aircraft Wing Using Carbon Fiber Reinforced Polymer and Glass Fiber Reinforced Polymer [Paper]. IOP Conference Series: Materials Science and Engineering, Volume 402, $2^{\text {nd }}$ International Conference on Advances in Mechanical Engineering (ICAME), Kattankulathur, India. https://doi.org/10.1088/1757899X/402/1/012077

León, C., \& Vásquez, A. (2010). Diseño y construcción de un soporte de ametralladora para el helicóptero del Ejército ecuatoriano, MI-171, utilizando materiales compuestos laminados [Tesis de grado, Universidad de las Fuerzas Armadas ESPE]. Repositorio de la Universidad de las Fuerzas Armadas ESPE, http://repositorio.espe.edu.ec/handle/21000/2342

Levy, A., \& Hubert, P. (2019). Vacuum-bagged Composite Laminate Forming Processes: Predicting Thickness Deviation in Complex Shapes. Composites Part A, 126(August), 105568. https://doi. org/10.1016/j.compositesa.2019.105568

Liu, X., He, Y., Qiu, D., \& Yu, Z. (2019). Numerical Optimizing and Experimental Evaluation of Stepwise Rapid High-pressure Microwave Curing Carbon Fiber/epoxy Composite Repair Patch. Composite Structures, 111529. https://doi.org/10.1016/j.compstruct.2019.111529

Muralidhara, B., Babu, S. P. K., \& Suresha, B. (2019). Materials Today: Proceedings Utilizing Vacuum Bagging Process to Prepare Carbon Fiber/epoxy Composites with Improved Mechanical Properties. MaterialsToday: Proceedings, 27(3), 2022-2028. https://doi.org/10.1016/j.matpr.2019.09.051

Pero-Sanz Elorz, J. A., Fernández González, D., \& Verdeja, L. F. (2019). Structural Materials: Properties and Selection. Springer. https://doi.org/10.1007/978-3-030-26161-0

Picu, C., \& Ganghoffer, J.-F. (Eds.). (2020). Mechanics of Fibrous Materials and Applications: Physical and Modelinf Aspects (Vol. 596). Springer. https://doi.org/10.1007/978-3-030-23846-9

Prasad, N. E., \& Wanhill, R. J. H. (Eds.). (2017). Aerospace Materials and Material Technologies (Vol. 1: Aerospace Materials). Springer. https://doi.org/10.1007/978-981-10-2134-3 
Siddiquee, S., Gan Jet Hong, M., \& Mizanur Rahman, M. (Eds.). (2020). Composite Materials: Applications in Engineering, Biomedicine and Food Science. Springer. https://doi.org/10.1007/978-3-030-45489-0

Suzuki, Y., Cousins, D., Wassgren, J., Kappes, B. B., \& Stebner, A. P. (2017). Kinetics and Temperature Evolution During The Bulk Polymerization of Methyl Methacrylate for Vacuum-Assisted Resin Transfer Molding. Composites Part A: Applied Science and Manufacturing, 104(January), 60-67. https:// doi.org/10.1016/j.compositesa.2017.10.022

Vasiliev, V. V., Jones, R. M., \& Man, L. I. (2017). Mechanics of Composite Structures. CRC Press. https://doi. org/10.1201/9780203747858

Verma, N., Kumar, R., Zafar, S., \& Pathak, H. (2020). Vacuum-assisted Microwave Curing of Epoxy/carbon Fiber Composite: An Attempt for Defect Reduction in Processing. Manufacturing Letters, 24 , 127-131. https://doi.org/10.1016/j.mfglet.2020.04.010

Wang, J., Baker, A., \& Chang, P. (2019). Hybrid Approaches for Aircraft Primary Structure Repairs. Composite Structures, 207(January), 190-203. https://doi.org/10.1016/j.compstruct.2018.09.038

Zhu, G., Wang, S., Cheng, W., Ren, Y., \& Wen, D. (2020). Corrosion and Wear Performance of Aircraft Skin After Laser Cleaning. Optics and Laser Technology, 132(July), 106475. https://doi.org/10.1016/j. optlastec.2020.106475

Zimmermann, N., \& Wang, P. H. (2020). A Review of Failure Modes and Fracture Analysis of Aircraft Composite Materials. Engineering Failure Analysis, 115(September), 104692. https://doi.org/10.1016/j. engfailanal.2020.104692 